\title{
Médiévales
}

Langues, Textes, Histoire

63 | automne 2012

Philosophies morales

\section{Tératologie de la morale, ou de l'éthique au Moyen Âge}

The Teratology of Morality, or Ethics in the Middle Ages

\section{Emanuele Coccia}

\section{(2) OpenEdition \\ 1 Journals}

\section{Édition électronique}

URL : https://journals.openedition.org/medievales/6800

DOI : $10.4000 /$ medievales. 6800

ISSN : 1777-5892

\section{Éditeur}

Presses universitaires de Vincennes

\section{Édition imprimée}

Date de publication : 15 décembre 2012

Pagination : 13-26

ISBN : 978-2-84292-353-2

ISSN : 0751-2708

\section{Référence électronique}

Emanuele Coccia, «Tératologie de la morale, ou de l'éthique au Moyen Âge », Médiévales [En ligne], 63 | automne 2012, mis en ligne le 15 janvier 2015, consulté le 22 avril 2022. URL : http://

journals.openedition.org/medievales/6800; DOI : https://doi.org/10.4000/medievales.6800 
Médiévales 63, automne 2012, p. 13-26

Emanuele COCCIA

\section{TÉRATOLOGIE DE LA MORALE, OU DE L'ÉTHIQUE AU MOYEN ÂGE}

Chaque fois que l'on se penche sur une époque du passé pour en observer et décrire les mœurs, l'on risque d'être saisi d'un étrange vertige'. À travers ses mœurs une société montre sans fard son visage le plus vrai et le plus intime. Le savoir qui règle et norme ces mœurs - la «morale» - trahit son dernier secret, la vérité ultime et suprême de cette société, le savoir actif qui a été à l'origine de chaque geste, chaque intention, chaque haleine de vie en son sein, et le spectacle qu'elle ne cesse de contempler en existant, en agissant, en se regardant elle-même.

Soutenir la vision de ce spectacle de l'extérieur n'est pas facile : en contemplant cette vérité l'on s'expose à la même force qui a été matériellement responsable du mode d'être de la totalité des membres d'une société. Et pourtant, le charme de cette vision est tellement puissant que la quasi-totalité des sciences sociales ont été séduites par lui. En pariant sur le fait que si les hommes disparaissent, la vérité qu'ils contemplent en agissant peut continuer à vivre après leur mort, les sciences sociales se sont lancées depuis très longtemps dans la quête de cet étrange Graal épistémologique qu'est la morale. Elles ont essayé de la récupérer dans le savoir sur le monde et l'être des choses qu'une société accumule, comme cela a été le cas dans l'anthropologie ; elles ont pu penser que cette vérité morale ne s'exprime pas à la première personne du singulier, mais prend le visage inquiétant d'un sujet sans nom et sans lucidité, capable de vouloir le mal et qui ne supporte pas d'être conscient, comme dans la psychanalyse ; elles ont pu chercher cette vérité en lui refusant

1. Je tiens à remercier Iacopo Costa, Aurélien Robert, Jakob Heller et Barbara Carnevali pour leurs conseils avisés et leurs suggestions. Ma gratitude va doublement à Aurélien Robert, pour son aide généreuse dans l'opération de francificatio de ma contribution. 
le titre de science du Bien, comme cela a été le cas dans la sociologie ${ }^{2}$. Mais en s'interrogeant sur la forme et le fondement ultime d'une société, elles se sont toujours lancées dans la quête de ce savoir ultime, capable de dévoiler la raison pour laquelle tout homme a pu faire et a effectivement fait ce qu'il a fait, ce qu'on appelle morale ${ }^{3}$. Par conséquent, toute tentative de saisir la vérité éthique d'une époque, qu'elle se soit solidifiée en la théorisation consciente d'une « philosophie morale » ou qu'elle se soit fossilisée dans les traces des gesta des hommes disparus, n'obéit, au fond, qu'au désir d'entendre à nouveau la voix de cette étrange Muse, capable d'inspirer toutes les actions humaines, quel qu'en soit le sujet.

Capturer cette voix, ainsi que déterminer univoquement et précisément le lieu où elle parle, se révèle pourtant être une tâche presque insurmontable. Il semblerait que, dans toute époque, sa voix se laisse entendre en même temps en plusieurs lieux, sous plusieurs formes et en plusieurs sujets. Supposons, par exemple, que nous voulions savoir quelle est la vérité éthique qui s'est exprimée au XIII $^{\mathrm{e}}$ siècle : l'on pourrait conjecturer que la morale du siècle s'exprime dans sa forme majeure dans les commentaires universitaires à l'Éthique à Nicomaque. Et pourtant, limiter sa recherche à ce genre de textes serait oublier que d'autres formes de théorisation à l'intérieur des mêmes universités ont atteint ces mêmes vérités morales. Il faudrait alors changer de point de vue, en reconnaissant que d'autres disciplines que la «philosophie morale » proprement dite (la médecine, la politique, la rhétorique, la théologie, la psychologie, l'anthropologie et aussi la littérature, si l'on en croit la lettre de Dante à Cangrande ${ }^{4}$ ) ont été des formes de production et de promotion d'un savoir éthique, qui reste tel même s'il ne se donne pas ce nom. Ce déplacement disciplinaire démontrerait alors que l'énonciation de vérités éthiques appartient toujours de droit à plusieurs disciplines et qu'une vérité éthique est toujours une vérité diffuse, existant toujours en dehors de la discipline qui lui donne son nom.

Mais la multiplicité des voix ne s'arrête pas là : il y aurait en effet un deuxième déplacement à accomplir, celui qui conduit des salles des universités

2. Pour les dettes des sciences sociales envers la tradition morale (surtout des moralistes français), cf. J. HeILBRon, Het ontstaan van de sociologie, 1990, Naissance de la sociologie, tr. fr. P. Dirk, Marseille, 2006, surtout p. 93-109, et B. Carnevali, « Mimésis littéraire et connaissance morale : la tradition de l'éthopée », Annales. Histoire, Sciences Sociales, 65 (2010), p. 291-322.

3. Cf. AL-Fāeāāi, De scientiiis, V (éd. F. Schupp, Hambourg, 2005, p. 112), qui parle d'une science qui « inquirit de speciebus operationum et consuetudinum voluntariarum, et de habitibus, et moribus, et segea, et gestibus, a quibus sunt ille actiones et consuetudines, et de finibus propter quos fiunt, et qualiter oportet ut sin tilla inventa in homine, et qualis sit modus ad comprehendendum et ordinandum ea in eis ».

4. Dante Alighieri, Epistola a Cangrande, XIII, 40 (éd. E. CeCChini, Florence, 1995) : « Genus vero philosophie, sub quo hic in toto et parte proceditur, est morale negotium, sive ethica ; quia non ad speculandum, sed ad opus inventum est totum et pars ». 
à d'autres lieux de production de la vérité morale : pour ne nommer que les plus évidents, il faudrait se pencher sur les cours, royales, princières et pontificales, sur les monastères et sur les familles, mais aussi prendre en considération la prédication et les cadres de la formation professionnelle qui ont développé diverses sortes de morales « déontologiques » liées aux métiers et aux différentes formes de vies (prière et contemplation, guerre, politique, etc.) $)^{5}$. Car l'éthique (au Moyen Âge comme dans toute autre époque et à la différence d'autres formes de savoir comme la métaphysique) est un savoir partagé par et diffusé parmi les acteurs sociaux les plus différents; elle a souvent pour objet non pas la totalité des hommes mais des microsociétés distinctes ; elle se multiplie et se façonne donc différemment en fonction des situations et des sujets qu'elle norme. Les exemples insérés dans le genre littéraire du Speculum principis, les règles de vie monastiques, les manuels d'amour (comme le De amore d'André le Chapelain) et les traités de civilité montrent que, loin de s'incarner exclusivement dans un énoncé universel qui exprimerait la nature de l'homme dans sa généralité, la réflexion éthique se traduit par une pluralité de codes particuliers et locaux, indépendants les uns des autres et capables d'exprimer seulement la vérité d'une situation et d'une forme de vie sui generis. S'il y a une pluralité de disciplines capables d'énoncer une vérité éthique, c'est parce que la prétendue vérité morale ultime concerne une pluralité de sujets et s'incarne dans une multiplicité d'objets (de manières de vivre) qui ne se laissent pas réduire moralement à un dénominateur commun.

Au Moyen Âge en outre, dans la voix de la Muse résonne toujours un fond plus grave : tous les savoirs moraux ainsi que toutes les théories éthiques partagent en effet une matrice épistémologique commune, et doivent s'inscrire dans le lieu ultime et suprême de toute doctrine et donc de toute vérité, l'Écriture sainte. Car, comme le dit Roger Bacon, « la science complète de cela est l'Écriture Sainte ${ }^{\dagger} »$. En ce sens, toute vérité éthique doit exister au moins en deux lieux épistémologiques à la fois : dans la théorie qui l'exprime et la formule « scientifiquement », mais aussi dans la Bible qui la fonde et lui donne une valeur normative universelle.

Loin de résonner dans la voix sibylline d'une Muse lucide sur ellemême qui recueillerait en elle l'Esprit du temps, la morale ressemble donc plutôt à une sorte d'hydre épistémologique qui, à travers ses multiples bouches, énonce des vérités dissonantes non seulement quant à leur message mais aussi dans leur forme et leur nom. Car le visage toujours différent qui les prononce répond à des noms de discipline différents (philosophie, éthique, règle, loi, conseil, etc.). Or cette capillarité, cette étrange capacité à se dédoubler, à se

5. Sur cet aspect, $c f$. le chef-d'œuvre d'A. Borst, Lebensformen im Mittelalter, Berlin, 1973.

6. Roger Bacon, Moralis Philosophia, V, 1 (éd. E. Massa, Zürich, 1953, p. 249) : « completa scientia de his est Sacra Scriptura ». 
déplacer, à être énoncée et théorisée par plusieurs sciences, à être professée par plusieurs acteurs sociaux d'un même contexte et à exister sous des formes épistémologiques différentes, constitue la qualité de la vérité éthique à tout moment. Depuis Nietzsche l'on est habitué à considérer la morale comme quelque chose de monstrueux: mais il s'agit d'une monstruosité épistémologique, celle d'une vérité qui existe toujours aussi hors du lieu dans lequel elle a été énoncée.

Cette dissonance dissimulée sous l'apparence d'une unité est en réalité la véritable differentia specifica de la vérité éthique, l'élément qui la distingue des autres vérités, d'ordre technique ou métaphysique. Pour l'énoncer de façon dogmatique l'on pourrait dire qu'une vérité éthique doit toujours être prononcée et connue aussi en dehors de sa discipline (elle est à la fois disciplinaire et hors disciplinaire), doit être partagée par ce qui ne la comprend pas (c'est ce que l'on appelle obéissance) et enfin, doit toujours pouvoir être énoncée selon plusieurs degrés de complexité et d'universalité. Exactement comme dans le mythe, celui qui tranche l'une de ses têtes en verra toujours pousser deux nouvelles.

\section{Droit/Morale}

En dépit de tout ce que l'on peut imaginer, la morale semblerait donc imposer au «Vrai » une étrange et dangereuse pullulation, soit sur le plan épistémologique, soit sur le plan social. Cette multiplication se fait encore plus radicale lorsque la vérité morale prétend assumer le nom de loi. Les relations de voisinage et d'intimité qui ont lié, à toute époque, la vérité morale à la vérité juridique ou politique ont toujours été très difficiles à comprendre. Le fait qu'un juriste renommé comme Hans Kelsen ait eu besoin, au $\mathrm{Xx}^{\mathrm{e}}$ siècle, de refuser explicitement toute inclusion du droit dans la morale ${ }^{7}$ pour distiller une essence pure de la norme, n'est en réalité que la preuve de la difficulté de séparer les deux « disciplines ». Sa revendication d'une autonomie du droit (de même que sa quête d'une jurisprudence épurée de toute relation avec la morale) est d'ailleurs loin d'être originale : d'un certain point de vue, elle n'est que la

7. H. KeLSEN, Reine Rechtstheorie. Einleitung in die rechtswissenschaftliche Problematik,

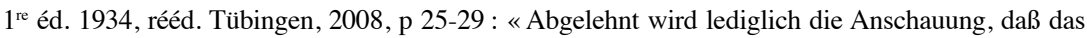
Recht als solches Bestandteil der Moral, daß also jedes Recht, als Recht, in irgendeinem Sinne und in irgendeinem Grade moralisch sei. Indem man das Recht als ein Teilgebiet der Moral darstellt, und dabei im Dunkeln läßt, ob dies nur die selbstverständliche Forderung bedeutet, daß das Recht moralisch gestaltet werden solle, oder ob damit gemeint ist, daß das Recht als Bestandteil der Moral tatsächlich moralischen Charakter habe, versucht man, dem Recht jenen absoluten Wert zu verleihen, den die Moral in Ansprucht nimmt. [...] Gegen sie [diese ideologischen Tendenzen, E.C.] ist die Reine Rechtstheorie gerichtet. Sie will das Recht darstelle, so wie es ist, ohne es als gerecht zu legitimieren oder als ungerecht zu disqualifizieren, sie fragt nach dem wirklichen und möglichen, nicht nach dem richtigen Recht. Sie ist in diesem Sinn eine radikal realistische Rechtstheorie. » 
version moderne de l'ancien Streit der Fakultaten éclaté au sein de l'université médiévale entre canonistes, artistes et théologiens ${ }^{8}$. Mais 1'histoire du droit (comprise comme la succession concrète et temporelle de la codification juridique), ainsi que l'histoire de la réflexion morale en Europe, comptent plus d'une tentative de démontrer ou de pratiquer l'équivalence entre le vrai juridique et le vrai moral. Au début de son Éthique à Nicomaque, Aristote affirmait déjà l'identité absolue entre la science morale et l'ensemble du savoir politique. Au Moyen Âge, on voyait la preuve de cette identité dans le texte biblique. Comme le dit Roger Bacon, « ce qu'Aristote appelle science morale est appelé par d'autres science politique, parce qu'elle démontre les droits des citoyens et des cités ${ }^{9} \gg$. Et les trois parties dont se compose la science morale coïncident avec les trois parties du Code de Loi par excellence, la Bible ${ }^{10}$.

Ce n'est pas une question purement nominale : ce partage de nom et de structure a suggéré à certains une approche réductrice. Ainsi, pour ne mentionner que l'exemple le plus frappant, Samuel Pufendorf a essayé de fonder « ontologiquement » le droit en ajoutant à la réalité des entia naturalia celle des entia moralia, dont le droit définirait l'ordonnancement ${ }^{11}$. Mais même

8. Cf. par exemple Henricus de Segusio, Summa una cum summariis et adnotationibus N. Superati, Lyon, 1537, c. 3v. : «Cui parti philosophiae supponatur. Ethice, id est morali, quia tractat de moribus, sicut et ceteri libri iuris [...], ut clericorum mores et per totum et nihilominus potest supponi et rationali, quia docet ornate et ordinate loquendi [...] sed et videtur quod naturali supponatur, quia tractat de naturalibus [...]. Sed hoc ultimum incidens est, duo vero prima principaliter insunt huic scientiae et civili quare haec scientia potest dici mixta quia partim rationalis vel partim moralis; nam et civilis scientia videtur sub se continere rhetorica quae rationalis est, unde Tullius in prima rhetorica post premium civilis uqaedam ratio est que multis et magnis ex reubus constat; et quedam magna et ampla pars est artificiosa eloquentia quam rhetoricam vocat etc. »

9. Roger Bacon, Moralis Philosophia, I, Prohoemium, p. 6 : « Haec vero scientia moralis vocatur ab Aristotelis et aliis civilis scientia, quia iura civium et civitatum demonstrat. Et quoniam solebant civitates dominari regionibus ut Roma imperabat mundo, ideo haec scientia civilis denominatur a civitate, iura tamen regni et imperii construendo. Haec autem scientia primo docet componere leges et iura vivendi; secundo docet ea credi et probari et homines excitari ad operandum et vivendum secundum illas leges.»

10. Ibid.: « Prima pars dividitur in tres : nam primo naturaliter occurrit ordinatio hominis in Deum, et respectu substantiarum angelicarum, secundo ad proximum, tertio ad seipsum, sicut Scriptura facit. Nam primo in libris Moysi sunt mandata et leges de Deo et cultu divino ; secundo de comparatione hominis ad proximum in eisdem libris et sequentibus ; tertio docetur de moribus, ut in libris Salomonis. Similiter in novo testamento hec tria tantummodo continetur ; nam homo non potest alias recipere comparationes. »

11. Samuel Pufendorf, De jure naturae et gentium, L. I, 3 (éd. F. BöHling, Berlin, 1998, p. 4) : «Exinde commodissime videmur entia moralia posse definire, quod sint modi quidam, rebus aut motibus physicis superadditi ab entibus intelligentibus, ad dirigendam potissimum et temperandam libertatem actuum hominis voluntariorum, et ad ordinationem aliquem et decorem vitae humanae conciliandum. [...] Ex hoc genere quoque sunt entia moralia. Quorum primum autorem merito Deum O. M. dixeris qui utique noluit, ut mortales hanc vitam sine cultu, sine more, ad brutorum instar transirent, sed ut ista et eorundem actiones certa ratione forent temperata : id 
chez les auteurs qui ne songent pas à réduire le droit à la morale, la question de la différence ou de l'identité entre les deux « disciplines » est plutôt difficile à résoudre. Les raisons de cette difficulté sont objectives. Il suffit de penser au concept stoïcien de kathekon (officium), ou aux réflexions cicéroniennes sur l'honestum (qui ont profondément influencé la tradition moderne), pour comprendre que déjà au niveau des concepts fondamentaux les deux disciplines sont très souvent confondues. Dans cet espace donc, les limites épistémologiques sont tellement faibles qu'il est parfois difficile de savoir si l'on a affaire à une norme juridique ou à une vérité d'ordre purement moral. Cette ambiguïté peut définir la nature même d'une norme, comme c'est le cas dans le corpus de règles monastiques, techniquement définies comme des recueils de consilia (des exhortations d'ordre moral), parfois appelés quasi iura, comme chez Humbert de Romans ${ }^{12}$, qui peuvent être transformés en droit positif à travers le vœu, comme le théorise Bernard de Clairvaux ${ }^{13}$. Une règle de vie démontre par son existence même que ce qu'on appelle droit n'est qu'une théorie morale que l'on a prise plus au sérieux que d'autres et que ce que l'on entend par « morale » n'est qu'une loi qui attend d'être promulguée.

Cette proximité ambiguë entre vérité éthique et vérité juridique ne produit pas seulement la confusion épistémologique entre les deux disciplines. Elle existe aussi comme une tension immanente au corps de la loi, dans la séparation rhétorique - si fréquemment pratiquée et théorisée dans le monde antique - entre les preambula legis, les prologues de la loi, c'est-à-dire la partie introductive rédigée à des fins d'exhortation et de persuasion (peistikos nomos) où le législateur énonce les principes moraux qui inspirent la norme, et le corps véritable de la norme, la loi pure (akratos nomos), qui contient l'élément soidisant purement juridique ${ }^{14}$. À partir de la longue réflexion sur les prologues de la loi, la tradition platonicienne avait enseigné à penser la relation entre morale

quod citra entia moralia fieri nequibat. Pleraque tamen arbitrio ipsorum hominum post superaddita, prout vitae humanae excolendae et velut in ordinem dirigendae, talia introduci proficuum videbatur. Hinc etiam finis eorundem patescit, qui non est, uti entium physicorum, perfectio hujus universi, sed peculiariter perfectio vitae humanae, quatenus prae brutorum vita decori cujusdam ordinis capax erat, utque in re maxime vaga, qualis est motus animi humani, concinna aliqua harmonia inveniretur. »

12. B. Humbertus de Romanis, Expositio super constitutiones fratrum praedicatorum, dans Opera de vita regulari, éd. J.-J. BERTHIER, vol. II, Rome, 1889, p. 20 : « Regulae vel consuetudines sunt quasi iura.»

13. Bernard de Clairvaux, Le Précepte et la Dispense, éd. H. Talbot, Paris 2000. Sur le rapport entre précepte et conseil, $c f$. J. HRUSCHKA, «Supererogation and Meritorious Duties », Jahrbuch für Recht und Ethik/Annual Review of Law and Ethics, 6 (1999), p. 93-108; D. HEYD, Supererogation : Its Status in Ethical Theory, Cambridge, 1992 ; C. CASAgrande, C. CRISCIANI et S. VeCCHIO éd., Consilium : teorie e pratiche del consigliare nella cultura medievale, Florence, 2004.

14. G. RIEs, Prolog und Epilog in Gesetzen des Altertums, Munich, 1983 ; H. Hunger, Prooimion. Elemente der byzantinischen Kaiseridee in den Arengen der Urkunden, Wien, 1964 ; M.-T. FöGEN, Das Lied vom Gesetz, Munich, 2008. 
et droit comme une différence rhétorique et non de nature. Selon Lycurgue par exemple, élève de Platon et d'Isocrate, la distance entre norme juridique et éducation morale, entre prescription et persuasion est surtout une question de brièveté :

Les lois, en effet, dans leur concision, n'enseignent pas, mais prescrivent la conduite à tenir, tandis que les poètes, en imitant la vie humaine, et en choisissant les plus beaux épisodes, persuadent les esprits, car ils développent leurs raisons et les illustrent par des exemples ${ }^{15}$.

La loi ne serait qu'une forme abrégée de l'enseignement moral ${ }^{16}$, et son caractère obligatoire serait à la fois le symptôme et la conséquence de cette nécessaire rapidité dans l'expression. Selon la perspective représentée dans cette tradition, la vérité morale existerait donc sous la forme la plus pure dans la loi et, en ce sens, ce serait dans la loi que l'historien devrait aller chercher l'expression suprême de la théorie morale d'une société à une époque déterminée.

\section{Prologues}

L'idée d'une continuité rhétorique et substantielle entre vérité morale et vérité juridique a trouvé son expression la plus radicale dans le milieu platonisant du judaïsme alexandrin. Mais c'est encore cette idée qui fondera la réflexion théologique du Moyen Âge latin. Dans ce cas, en effet, la continuité ou plutôt la coïncidence rhétorique et presque physique entre droit et morale n'est pas le résultat d'une théorie, mais une évidence textuelle et culturelle qu'il faut pouvoir justifier ex post. En reconstruisant l'histoire du droit européen, l'on oublie souvent, en effet, que le premier grand exemple de codification juridique à s'être matérialisé dans la Méditerranée hellénistique n'est pas le Corpus juris civilis de Justinien, mais le Tanakh juif, dans sa version grecque, la Septante. C'est littéralement comme «Livres de la loi des juifs » (tou nomou tôn ioudaiôn biblia) que ce texte est appelé dans le premier document qui le nomme, la Lettre à Aristée, qui justifie la traduction en grec du texte par la volonté de compléter la collection juridique de la Bibliothèque d'Alexandrie ${ }^{17}$. Et c'est en tant que plus ancien code de loi de l'histoire que la

15. Lycurgue, Contre Léocrate, $\S 102$ (éd. et tr. fr. F. Durrbach, Paris, 1971, p. 67).

16. Platon, Nomoi 721 a (ID., CEuvres complètes. Tome XI. $2^{e}$ Partie. Les Lois, Livres III-VI, éd. et tr. fr. É. DES Places, Paris, 1951, p. 74) : «il faut toujours préférer la brièveté (ta brachutera)».

17. Lettre d'Aristée à Philocrate, IV, 30 (éd. et tr. fr. A. Pellettier, Paris, 1962, p. 118-120 et p. 125-127) : «Sur ton ordre, ô roi, en ce qui concerne les ouvrages qui manquent encore, et qu'il faut réunir pour compléter la Bibliothèque, et la réparation de ceux qui sont en mauvais état, j'y ai mis tous mes soins et je viens te soumettre le projet suivant : outre quelques autres, les livres de la loi des Juifs (tou nomou tôn ioudaiôn biblia) nous manquent, car ils se lisent en caractère et prononciation et ont été écrits avec assez de négligences et d'inexactitudes, au dire des hommes 
Torah est considérée dans le deuxième livre du traité apologétique de Flavius Josèphe, Contre Apion, qui dresse une sorte d'étude comparative des formes de législation antiques. Moïse, selon Flavius, est «le plus ancien des législateur connus du monde entier » :

Les Lycurgue, les Solon, les Zaulecos de Locres, et tous ceux qu'on admire chez les Grecs paraissent nés hier ou d'avant-hier comparés à lui, puisque le nom même de loi dans l'Antiquité était inconnu en Grèce. Témoin Homère qui nulle part dans ses poèmes ne s'en est servi. En effet la loi n'existait même pas de son temps ; les peuples étaient gouvernés suivant des maximes non définies et par les ordres des rois (gnômais aoristois ta plêthê diôkeito kai prostagmasi tôn basileôn). Longtemps encore ils continuèrent à suivre des coutumes non écrites (ethesin agraphois) dont beaucoup, au fur et à mesure des circonstances, étaient modifiées ${ }^{18}$.

Si la loi des juifs est si ancienne qu'elle a «ouvert la voie de la vie légale (to zên nomimôs) » ${ }^{19}$ aux autres peuples, il faut bien reconnaître que ses particularités textuelles et rhétoriques la rendent incomparable avec toute autre forme de législation produite dans le monde grec. Au lieu de penser une articulation interne entre un élément non juridique et un élément proprement normatif à l'intérieur de la loi, comme c'était le cas dans les théories sur le prologue de la loi, ici, c'est une théorie de la pluralité des registres de la loi qui se développe. Selon cette nouvelle "poétique juridique », la loi ne coïncide pas avec le commandement parce qu'elle ne parle pas seulement le langage du précepte $^{20}$. Elle s'exprime aussi à travers d'autres genres rhétoriques, comme l'histoire, la biographie, le conte, etc. :

Les oracles dont le prophète Moïse fut l'instrument sont de trois genres (ideai) : l'une concerne la création, l'autre est historique, la troisième législative (nomothetikê). [...] Quant à la partie historique, c'est la relation de vies

compétents : il leur a manqué une sollicitude royale (pronoia basilikê). Or il faut que ces livres aussi tu les aies, dans un texte correct, car cette loi est pleine de sagesse et très pure, puisqu'elle est divine (dia to kai philosophôteran einai kai akeraion tên nomothesian tautên, hôs an ousan theian)». Plus avant (Lettre du Roi Ptolémée à Éléazar) : « Désireux de leur être agréable, ainsi qu'à tous les juifs de la terre et à leurs descendants, nous avons décidé de faire traduire votre Loi de ce que vous appelez le texte hébreu en langue grecque pour avoir ces livres-là aussi dans notre bibliothèque, avec les autres "livres du Roi". Dans ces conditions tu ferais bien et tu répondrais à notre sollicitude, en choisissant des hommes d'une vie exemplaire, des Anciens versés dans la connaissance de leur Loi (presbuterous empeirian echontas tou nomou), capables d'en faire une traduction, six de chaque tribu, pour trouver un texte qui représente l'accord de la majorité, vu l'importance de la recherche. »

18. Flavius JosèPhe, Contre Apion, L. II, chap. XV, § 154-155 (éd. et tr. fr. T. Reinach et L. BLuM, rééd. Paris, 2003, p. 84).

19. Ibid.

20. Philon D’Alexandrie, De opificio mundi, § 1-2 (éd. et tr. fr. R. Arnaldez, Paris, 1961, p. 145). 
vertueuses et de vies mauvaises (anagraphê bion esti spoudaiôn kai ponêrôn), avec les peines et récompenses ayant sanctionné les unes et les autres en chaque génération ${ }^{21}$.

\section{En développant cette même idée, Flavius avait remarqué que}

toute instruction et toute éducation morale peuvent, en effet, se faire de deux manières : par des préceptes qu'on enseigne, ou par la pratique des mœurs (dia tês askêseôs tôn ethôn). Les autres législateurs ont différé d'opinion et, choisissant chacun celle des deux manières qui leur convenait, ont négligé l'autre. Par exemple les Lacédémoniens et les Crétois élevaient les citoyens par la pratique, non par des préceptes. D'autre part, les Athéniens et presque tous les autres Grecs prescrivaient par les lois ce qu'il fallait faire ou éviter, mais ne se souciaient point d'en donner l'habitude par l'action. Notre législateur, lui, a mis tous ses soins à concilier ces deux enseignements ${ }^{22}$.

Si la biographie et l'histoire deviennent ainsi l'une des formes de cette nouvelle jurisprudence, il est bien évident que, dans ce contexte, la vérité morale - comme la vérité juridique - ne se laissera plus reconnaître par son lieu d'énonciation ou par sa forme rhétorique.

La pluralité des registres et le dépassement de la simple forme du commandement n'expriment pas un manque de la loi, ni un défaut de sa nature juridique. Bien au contraire, ils marquent la perfection et la supériorité de la Loi juive sur toute autre forme normative. En développant des idées déjà formulées dans le traité Sur les Lois de Platon $\left[719^{\mathrm{b}}-723^{\mathrm{e}}\right]$, Philon considère la coïncidence linguistique et rhétorique du droit et de la morale, de l'exhortation et du commandement, comme le signe de la perfection absolue de l'activité d'un législateur :

Parmi les législateurs, les uns, ayant tout de suite établi la liste des choses à faire et à ne pas faire, ont fixé les peines pour les contrevenants, les autres, s'estimant supérieurs, n'ont pas commencé par là, mais, ayant d'abord fondé et établi une

21. ID., De praemiis et poenis, § 1-2 (éd. et tr. fr. A. BECKAERT, Paris, 1961, p. 42, traduction légèrement modifiée).

22. Flavius JosÈPHe, Contre Apion, chap. XVI, § 170-171 (p. 86-88). Le texte continue ainsi : «Il n'a point laissé sans explication la pratique des mœurs, ni souffert que le texte de la loi fût sans effet ; à commencer par la première éducation et la vie domestique de chacun, il n'a rien laissé pas même le moindre détail à l'initiative et à la fantaisie des assujettis; même les mets dont il faut s'abstenir ou qu'on peut manger, les personnes qu'on peut admettre à partager notre vie, l'application au travail et inversement le repos, il a lui-même délimité et réglé tout cela pour eux par sa loi, afin que vivant sous elle comme soumis à un père et un maître, nous ne péchions en rien ni volontairement ni par ignorance. Car il n'a pas non plus laissé l'excuse de l'ignorance ; il a proclamé la loi l'enseignement le plus beau et le plus nécessaire [kai kalliston kai anagkaiotaton apeidexe paideuma ton nomon] ; ce n'est pas une fois, ni deux ni plusieurs qu'il faut l'entendre : mais il a ordonné que chaque semaine, abandonnant tous autres travaux on se réunit pour écouter la loi et l'apprendre exactement par cœur. C'est ce que tous les législateurs semblent avoir négligé » (ibid., chap. XVII, § 173-175, p. 88-90). 
cité par le raisonnement, ils ont, par la création des lois, adapté à la cité ainsi fondée la constitution qu'ils jugeaient le mieux en rapport et la plus adéquate ; mais Moïse ayant estimé que la première attitude, celle qui consiste à enjoindre sans encourager comme si l'on s'adressait non à des hommes libres mais à des esclaves, est le fait d'un tyran et d'un despote - ce qu'elle était effectivement - et d'autre part que la seconde, bien qu'appropriée à son objet, ne semblait pas néanmoins avoir obtenu l'approbation complète de tous les juges, Moïse, dis-je, adopta une attitude différente, dans chacun des deux secteurs.

D'une part, dans ses ordres et ses interdictions il suggère et encourage plutôt qu'il ne commande, s'efforçant d'accompagner d'introductions et de conclusions la plupart des instructions indispensables qu'il donne pour incliner par consentement plutôt que par violence. D'autre part, ayant jugé au-dessous de la dignité des lois de commencer sa rédaction par la fondation d'une cité faite de main d'homme, ayant pris une vue d'ensemble de la grandeur et de la beauté de tout le code grâce au regard précis de son intelligence, et ayant estimé qu'il était trop puissant et trop divin pour être renfermé dans des limites terrestres, il commença par la Genèse de la Grande Cité dans la pensée que les lois étaient l'image la plus ressemblante de la constitution de l'univers ${ }^{23}$.

Le monde chrétien fait face à une situation culturelle et textuelle tout à fait semblable: le code juridique par excellence au Moyen Âge, celui qui fonde toute autre forme de droit et qui rend possible la justice sur terre, l'Écriture sainte, laisse coïncider dans un même texte un registre de préceptes, plus approprié à un code de loi, et des registres littéraires ou de parénèse, typiques de l'édification morale. Dans la Bible, en effet, vérité morale et vérité juridique se confondent sans reste : penser la Bible et théoriser l'existence de l'Écriture sainte a donc représenté, pour le Moyen Âge, le défi de penser, de théoriser la proximité ou l'identité entre droit et morale, entre vérité éthique et vérité juridique. C'est seulement à partir de la considération de ces évidences, qu'on peut lire (et relativiser) la revendication des « philosophes » à propos de l'autonomie du savoir moral et de son unicité. Au Moyen Âge, ainsi qu'à toute autre époque, la morale existe surtout là où les philosophes se taisent.

\section{Vie et norme}

Il ne revient pas seulement à la Bible de sceller la nature morale de toute vérité juridique. Si toute norme - et donc toute vérité juridique - n'est au fond qu'une vérité morale, c'est parce que la loi suprême, avant de s'exprimer en mots dans la Nouvelle Loi, a existé comme mos, coutume, forme de vie singulière, celle du Christ. L'idée du Christ comme lex animata, qui aurait en quelque sorte transformé ses mœurs, sa vie, sa propre «morale » en loi, qui

23. Philon D’AleXandrie, De vita Mosis, II, § 49-53 (éd. et tr. fr. R. Arnaldez, C. MondéSERT, J. Pouilloux et P. SaVinel, Paris, 1967, p. 213-215). 
n'est autre que la loi suprême que l'on peut lire dans les Évangiles, s'est imposée très tôt dans la tradition théologique chrétienne ${ }^{24}$ et constitue l'origine lointaine de la tentative dans la tradition monastique de penser l'identité entre vie et norme ${ }^{25}$. Dans une certaine mesure, toute théorie morale n'est qu'une traduction plus ou moins immédiate des vérités incarnées dans et exemplifiées par cette vie primordiale et paradigmatique, racontée par les quatre biographies officielles incluses dans le canon néotestamentaire. Ici aussi, l'hydre ne cesse de multiplier ses têtes : s'il y a une vérité morale « scientifiquement formulée », elle existe toujours à côté de celle littérairement exprimée dans les Évangiles, et elle doit se penser comme équivalente à ces contes, sans pouvoir vraiment rivaliser avec eux. Si le projet d'un droit pur n'est qu'un rêve pour universitaires, les projets pour produire une morale pure sont également destinés à échouer. Les vérités morales sont toujours très instables : elles ne résistent pas longtemps à la transformation, elles ne restent jamais telles qu'elles sont. Pour exister ou pour se conserver, elles doivent changer leur nature : devenir vérités juridiques, littéraires, coutumières. La morale est ce qui donne au «vrai » la capacité de se métamorphoser.

Mais la logique de l'incarnation n'épuise pas la vie du « vrai » dans la morale. Car s'il est vrai qu'une théorie éthique ne peut jamais exister seulement comme théorie, et qu'elle doit aussi se réaliser dans un acte, il reste également vrai que ce dédoublement ne se produit jamais sous le signe de l'identité comme c'est le cas dans d'autres formes de vérité. Une vérité éthique doit pouvoir se redoubler dans la vie d'un individu qui l'applique, mais l'absence de l'application ou la genèse, dans la vie concrète, d'une autre vérité n'annule pas la validité, la vérité et l'autonomie de la première : l'existence et la Geltung d'une norme n'est pas du tout mise en danger par le fait de n'avoir pas été respectée, ni par le fait d'avoir été dédoublée dans la praxis par une autre vérité. Cela peut sembler paradoxal, mais dans la morale le vrai développe une tolérance extrême au dédoublement, à la multiplication, à la dualité entre théorie et pratique. Une théorie éthique doit présupposer la possibilité de ne

24. Lactance, Divinae institutiones, IV (éd. et tr. fr. P. Monat, Paris, 1992, p. 190-192) : «Quicumque praecepta dat hominibus ad vitam moresque fingit aliorum, quaero debeatne ipse facere quae praecipit an non debeat. Si non fecerit soluta praecepta sunt. Si enim bona sunt quae praecipiuntur si vitam hominum in optimo statu collocant, non se debet ipse praeceptor a numero coetuque hominum segregare inter quos agit, et ipsi eodem modo vivendum est quo docet esse vivendum, ne, si aliter vixerit, ipse praeciptis suis fiem detrahat leviorem doctrinam suam faciat, si re ipsa resolvat quod verbis nitatur adstingere. Unusquisque enim cum audit praecipientem non vult imponi sibi necessitate parendi, tamquam sibi ius libertatis adimatur. [...] Homines enim malunt exempla quam verba quia loqui facile est praestare difficile. »

25. Étienne de TournaI, Epistula LXXI à Robert de Pontigny (ID., Epistulae, éd. J.-P. Migne, PL 211, col. 368) : «Libellus etiam qui eorum constitutiones continet, non regula appellatur ab eis, sed vita. Inde est quod sicut regula quam observant canonici nostri dicuntur regulares, sic etiam ipsi necesse est a vita quam profitentur dicantur clerici seu laici vitales. » 
pas se réaliser dans la pratique, elle est même définie par cette possibilité. L'autonomie de la vérité morale est avant tout une autonomie par rapport à sa réalité concrète. C'est dans cette autonomie que réside la source de l'immortalité de cette hydre épistémologique que continue à séduire et à rendre impossible l'accomplissement des sciences sociales. Car son existence est la garantie qu'en morale la vérité ne pourra jamais parler d'une seule voix.

\section{Peuples}

Le jeu de dédoublement ne se produit pas seulement dans le rapport d'une vérité morale, avec un individu, mais aussi avec une multitude. Une théorie morale n'est en effet telle que si elle engage non seulement un individu mais une communauté, et même mieux une collectivité homogène : les vérités morales sembleraient donc devoir exister toujours comme mœurs collectivement partagées, en somme comme peuples réellement existants. Passer en revue des théories éthiques devrait signifier esquisser un tableau des peuples et des civilisations. Une science morale devrait donc coïncider tout court avec une théorie des sociétés, c'est-à-dire une sociologie, une anthropologie, ou mieux encore une sorte d'ethnographie ${ }^{26}$. C'est exactement dans cet esprit que, dans le troisième livre de sa Moralis Philosophia, Roger Bacon développe une sorte de phénoménologie des civilisations entendues comme des entités éthiques. Bacon explique que l'on peut classer toutes les sectae, c'est-à-dire les communautés définie par une lex (l'on pourrait traduire, par une vérité morale), en fonction de la manière dans laquelle elles conçoivent le bonheur, c'est-àdire le but qu'elles poursuivent ${ }^{27}$. Ce but, qui peut être simple ou complexe, peut consister dans le plaisir, la richesse, l'honneur, la puissance, la gloire, la félicité dans l'autre vie (dans les plaisirs du corps ou de l'âme) ou dans leur combinaison réciproque. Selon Bacon, les nations principales du monde (celles qui résisteront jusqu'à la venue de l'Antéchrist) sont au nombre de $\operatorname{six}^{28}$ : les

26. Ce sera la perspective de Lucien Lévy-Bruhl dans La Morale et la science des mœurs, Paris, 1903.

27. Cette tradition d'individuation morale des peuples culminera, comme l'on sait, dans le chef-d'œuvre de Montesquieu, De l'esprit des lois.

28. Roger BACON, Moralis Philosophia, IV, d. I, chap. I, p. 188-189 : « Aristotiles autem in sua Philosophia discendi ad species sectarum, et dicit quod ipse vult considerare de sectis et legibus civitatum quatuor vel quinque simplicium, et videre quae leges corrumpunt civitates et regna et quae non. Et dicit quatuor esse vel quinque semplice sextas corruptas, intendens quod secta vel lex dicitur simplex propter finem semplice et composita propter finem compositum quia omnis secta variatur secundum condicionem finis, ut docet Alfarabius. [...] Et isti fines simplices, secundum Alfarabium, et evidentius secundum Boethium tertio De consolatione, sunt voluptas, divitiae, honor potentia fama seu gloria nominis. Sectae autem compositae fiunt ex omnibus istis vel quatuor quibuscumque vel tribus vel duo bus secundum diversas combinationes. Sed praeter hos fines est alius, scilicet felicitas alterius vitae, quam diversi diversimode quaeruntet intendunt ; quia quidam ponunt hanc in deliciis corporis, quidam in deliciis animae, quidam in deliciis utriusque. 
sarraceni, qui cherchent les plaisirs de la vie terrestre (delectationes vitae praesentis $)^{29}$; les tartari, qui désirent seulement la puissance ${ }^{30}$; les païens qui pensent la félicité dans l'autre monde par analogie avec celle du monde présent $^{31}$; les idolatrae ${ }^{32}$, les juifs ${ }^{33}$ et enfin les chrétiens ${ }^{34}$. L'intérêt de ce tableau réside dans le fait que ces vérités morales (qu'elles soient considérées comme passions, vices ou vertus) deviennent des principes d'individuation ethnographique : elles sont capables de fonder la vie d'un peuple parce qu'elles tracent les limites des civilisations en leur assignant leur place dans le parcours historique de l'humanité. L'affirmation de la supériorité d'une vérité éthique sur les autres ne peut que coïncider avec la victoire d'un peuple (secta) sur tous les autres. L'histoire politique se transforme en quelque sorte en la bataille des vérités morales prononcées par la même hydre : c'est comme si ses têtes étaient constamment en guerre entre elles, comme si de la bouche de chacune d'elles se levaient des insultes envers les autres, comme si l'histoire n'était rien d'autre que la somme des tentatives, répétées et vaines, d'accorder ses multiples voix. On touche ici au paradoxe le plus curieux de la morale. Car au lieu de combattre

[...] Et ideo recitabo nationes principales apud quas variantur sectae, quae modo currunt per mundum ut sunt Saraceni, Tartari, Pagani, Idololatrae, Iudaei, Christiani : non enim sunt plures sectae principales nec possunt esse usque ad sectam Antichristi. »

29. Ibid., p. 189-190 : «Quidam autem volunt delectaciones vite presentis habere, non estimantes se deficere a futura felicitate, qualitercumque abutantur bonis temporalibus; et immergunt se illecebris voluptatum, ut sarraceni, qui uxores multiplicant quantum volunt secundum legem suam. »

30. Ibid., p. 190: «Quidam vero ardent libidine dominandi, ut tartari, secundum quod imperator eorum dicit unum dominum debere esse in terra, sicut est unus deus in celo. »

31. Ibid. : «Pagani vero puri, consuetudine vivendi pro racione legum utentes, ut Purceni et naciones confines eis, deliciis et diviciis et honore et huiusmodi istius vite detinentur cum intencione alterius, ut qualis fuerit hic et quantus talis et tantus estimatur fore in vita futura. Unde in morte faciunt se comburi publice cum lapidibus preciosis et auro et argento et familia et amicis et omnibus diviciis et bonis sperantes quod post mortem omnibus hiis gaudebunt. »

32. Ibid., p. 191 : «Similiter Ydolatre, cum bonis istius mundi credunt possidere futura, excepto quod sacerdotes eorum casitatem vovent et a delectacione luxurie gaudent abstinere, sicut patet ex regionibus orientis in parte aquilonari [...]. Et omnes isti expectant bona corporalia alterius vite, nichil de spiritualibus sapientes; et non est contra leges eorum, ut qualitercumque possunt, querant bona huius mundi, nec se reputant qualitercumque contingant, frustrari a vita eterna.»

33. Ibid. : «Set iudaei bona temporalia et eterna sperabant ; diversimode tamen, quoniam spiritualiter sapientes virtutem legis, aspirabant ad bona non solum corporis set anime ; litteraliter vero considerantes legem, credebant bona alterius vitae tantum corporalia. Similiter nec per fas et nephas, secundum legem eorum quaerunt temporalia, set auctoritatem Dei et secundum iura. Licet enim spoliaverunt multas naciones et subiugaverunt, hoc fecerunt secundum iusticiam. »

34. Ibid., p. 191-192: "Christiani vero spiritualibus spiritualia comparantes, secundum legem suam possunt temporalia habere propter humanam fragilitatem ut exerceant spiritualia in hac vita, quatinus tandem perveniant ad eterna tam corporaliter quam spiritualiter. Et tamen in illa vivent sine rebus extrinsecis, quibus in hac vita utuntur homines; nam corpus animale fiet spirituale, et totus homo glorificabitur et vivet cum deo et angeli sanctis. » 
ceux qui osent s'en approcher, il semblerait que cette hydre ne cesse de se combattre elle-même et de se rendre la vie impossible.

Emanuele CoccIa - CENJ-EHESS, 105 Bd Raspail, 75006 Paris

\section{Tératologie de la morale, ou de l'éthique au Moyen Âge}

L'article se concentre sur le statut épistémologique de la vérité morale: contrairement à d'autres formes de vérité, la vérité morale existe toujours dans des endroits autres que le lieu de sa formalisation dans des enseignements universitaires, car elle n'est jamais unique. Les identités morales d'une époque font l'objet de différentes sciences (le droit, l'éthique, la rhétorique, etc.), s'incarnent dans des figures et des contextes sociaux très différents et existent souvent sous des formes contradictoires. Toute tentative d'écrire une histoire de la pensée morale au Moyen Âge devrait donc dépasser l'analyse des commentaires sur l'Éthique ou de la production académique. Après une discussion générale de la question, l'article se concentre sur trois aspects : la relation entre vérité morale et vérité juridique à partir du problème des «prologues de la loi »; la façon dont le Nouveau Testament présente l'existence de la vérité morale dans le corps même du Christ, et, enfin, la façon dont certains théologiens pensèrent la relation entre l'unité de la vérité morale et la pluralité ethnique et politique des formes de vie.

Droit - Nouveau Testament - sciences sociales - épistémologie - prologues de la loi

\section{The Teratology of Morality or Ethics in the Middle Ages}

The article focuses on the peculiar epistemological status of moral truth: unlike other kinds of truth, moral truth does always exist in other places than the place of its formalization in academic teachings, and is never unique. The various moral identities of a specific period are the objects of different sciences (in the past: law, ethics, rhetoric etc.; nowadays anthropology, sociology, ethics etc.); they also express themselves in different contexts and do exist in contradictory forms. Every attempt of writing a history of moral thought in the Middle Ages should consider the epistemological and empirical plurality of moral truth and stop concentrating itself on the analysis of the commentaries on Ethics or on the academic production. After a general discussion of this problem, the article focuses on three questions: the relationship between moral truth and juridical truth as exemplified in the "preludes to law"; the way moral truth exists in the very body of Christ following the Gospels; and finally the way some theologians thought the relationship between the unity of moral truth and the ethnic and political plurality of human ways of life.

Right - New Testament - social sciences - epistemology - prelude to law 